\title{
Adolescents of the world: move!!!
}

\author{
Bruno Caramelli, ${ }^{1}$ Isabela Giuliano ${ }^{2}$
}

\begin{abstract}
$T_{\text {h }}$ hroughout human history adolescents have always been in the frontline of conflicts: soldiers in wars, protestors on the streets. Nowadays adolescents remain in the same position: they are the most numerous victims of social inequality, of a lack of prospects for the future, of unemployment, violence and drug abuse. In this issue of the Jornal de Pediatria, Kelishadi et al., ${ }^{1}$ in
\end{abstract} the name of the Isfahan Healthy Heart Program and the longitudinal intervention of the Heart Health Promotion from Childhood intervention, have added another factor to the list threatening the future of adolescents. The authors describe the elevated cumulative prevalence of cardiovascular risk factors among the adolescents of Iran. The researchers at the Isfahan Cardiovascular Research Center at the Isfahan University of Medical Sciences (a World Health Organization collaborative center) performed a cross-sectional study of 2,000 adolescents of both sexes aged 11 to 18 years, randomly recruited at the urban and rural zones of three cities in Iran.

Investigating Iranian adolescents, they observed that more than $79 \%$ were exposed to at least one and $24 \%$ to at least two cardiovascular risk factors (smoking, arterial hypertension, dyslipidemia, obesity or physical inactivity). The observed prevalence rates for physical inactivity and dyslipidemia are even more concerning if one considers that they may be underestimated because the reference values used are based on percentiles and not absolute figures. In Brazil, Giuliano et al. ${ }^{2}$ observed similar patterns among 1,053 children and adolescents aged 7 to 18 years in Florianopolis, Santa Catarina state and provided information on characteristics and the number of hours without daily physical activity. In that study, $42 \%$ of the individuals spent more than two hours per day on activities such as television or video games. In the study carried out by Kelishadi et al., more than $66 \%$ of the individuals were considered physically

1. Director of the Unidade de Medicina Interdisciplinar em Cardiologia do InCor-HCFMUSP. Associate professor, Universidade de São Paulo (USP), São Paulo, SP, Brazil.

2. PhD student, Department of Cardiopulmonology, Physician, USP, São Paulo, SP, Brazil.

Suggested citation: Caramelli B, Giuliano I. Adolescents of the world: move!!! J Pediatr (Rio J). 2005;81:427-8. inactive for fulfilling the defining criteria, namely watch television for more than 3 hours and not participate in at least three sessions per week lasting at least 20 minutes of steady exercise.

Diverse hypotheses have been offered to explain this phenomenon. The urbanization that has taken place in recent decades has obliged children to limit the time the can spend outside. As urban violence has increased, they have learned to spend their leisure time on sedentary activities such as television, electronic games and computers. Daily activities such as walking or riding bicycles to school have been abandoned in the name of safety and the justified fears of parents. The combination of these factors with the ever greater offer of a wide variety of foods with high calorie densities has resulted in an epidemic increase in the body mass index of children all over the world.

Iran is a country with a population distribution similar to Brazil, with a large number of young people at the base and middle of the pyramid and a life expectancy that has risen significantly in recent years, probably as a result of the decrease of infant mortality. This situation indicates that the two countries could suffer a significant increase in the incidence of cardiovascular diseases as these individuals begin to reach later life. Cross-sectional studies like the one by Kelishadi et al. are important for identifying the whole panorama of diseases such as cardiovascular ones. They have been at the top of cause of death lists for many years and have many characteristics that unfortunately make reversal of this situation very difficult. In contrast to infectious diseases and cancer, the most feared clinical manifestations of atherosclerosis (acute myocardial infarction and stroke) almost never have precursor signs or symptoms. A survey carried out by the WHO's MONICA project found that the majority of individuals who die from myocardial infarction do so within the first hours of the onset of symptoms, i.e. before it is possible to intervene medically. ${ }^{3}$ Under such conditions prevention must be the treatment of choice both on an individual and a population level. Efforts towards this end have been made in Brazil in recent years. ${ }^{2,4}$ The determination of those cardiovascular risk factors that are modifiable in the Iranian population by Kelishadi et al. can be of great help in development of specific strategies, aimed at the control and prevention of 
the disease. The identification of physical inactivity, the case in more than $66 \%$ of the individuals studied, constituting the most important factor in this sample, warns of the urgency of creating intervention proposals. In their article the authors recognize the limitations of their study for correctly identifying the existence of the phenomenon of risk factor clustering. Despite this, it is very likely that interventions aimed at physical inactivity can be associated with reductions in the prevalence of other factors like obesity, arterial hypertension and dyslipidemia.

If on one hand the advertised "explosion" in the incidence of cardiovascular diseases that may happen over the next few decades is a serious problem for health systems, on the other the lessons learnt from previous examples executed in other countries could be a strategic advantage. Undoubtedly they have been effective: nowadays the need for taxation and legislation on the use of cigarettes and alcohol are unquestioned, as are the value of dietary models, the food groups intake pyramid and publicity on the concept of a healthy diet. It is nevertheless debatable whether these strategies will be enough to combat what is predicted. A permanent state of vigilance must be put into practice so that intervention and monitoring studies can be rapidly implanted. This study by Kelishadi et al. ${ }^{1}$ represents just the first step in this direction. Indeed, the authors report that the study served as the basis for a major longitudinal intervention study called Heart Health Promotion from Childhood.
New strategies for intervening in the risk factors for cardiovascular disease proposed by the public health system or by private initiative and aimed at cardiovascular prevention for children and adolescents should be tested at an accelerated pace. Nevertheless, it is necessary to remember and respect the special characteristics of each population group. Furthermore, if the results of these studies by Kelishadi et al. ${ }^{1}$ and Giuliano et al., ${ }^{2}$ are considered, then, out of all the risk factors, physical activity is the one to aim for.

\section{References}

1. Kelishadi R, Sadri G, Tavasoli A, Kahbasi M, Roohafza H, Sadeghi $M$, et al. Cumulative prevalence of risk factors for atherosclerotic cardiovascular diseases in Iranian adolescents. J Pediatr (Rio J). 2005;81:447-53.

2. Giuliano I, Coutinho M, Freitas S, Pires M, Zunino J, Ribeiro R. Lípides séricos em crianças e adolescentes de Florianópolis - SC - Estudo Floripa Saudável 2040. Arq Bras Cardiol. 2005;85:85-91.

3. Chambless $L$, Keil $U$, Dobson A, Mähönen M, Kuulasmaa K, Rajakangas $A$, et al. Population versus clinical view of case fatality from acute coronary heart disease: results from the WHO MONICA Project 1985-1990. Circulation. 1997; 96:3849-59.

4. Magalhaes M, Pozzan R, Brandao A, Cerqueira R, Rousoulieres A, Szwarcwald C, et al. Early blood pressure level as a mark of familial aggregation of metabolic cardiovascular risk factors the Rio de Janeiro Study. J Hypertens. 1998;16(12 Pt 2):1885-9. 